\title{
Using a neural network to make operational forecasts of ionospheric variations and storms at Kokubunji, Japan
}

\author{
Maho I. Nakamura ${ }^{1,2}$, Takashi Maruyama ${ }^{1}$, and Yasunari Shidama ${ }^{2}$ \\ ${ }^{1}$ National Institute of Information and Communications Technology, Japan \\ ${ }^{2}$ Shinshu University, Japan
}

(Received May 30, 2007; Revised November 27, 2007; Accepted November 27, 2007; Online published January 11, 2008)

\begin{abstract}
An operational model was developed for forecasting ionospheric variations and storms at Kokubunji $\left(35^{\circ} \mathrm{N}\right.$, $139^{\circ} \mathrm{E}$ ), 24 hours in advance, by using a neural network. The ionospheric critical frequency (foF2) shows periodic variabilities from days to the solar cycle length and also shows sporadic changes known as ionospheric storms caused by geomagnetic storms (of solar disturbance origin). The neural network was trained for the target parameter of foF2 at each local time and input parameters of solar flux, sunspot number, day of the year, Kindex at Kakioka. The training was conducted using the data obtained for the period from 1960 to 1984 . The method was validated for the period from 1985 to 2003. The trained network can be used for daily forecasting ionospheric variations including storms using prompt daily reports of K-index, sunspot number, and solar flux values available on-line.
\end{abstract}

Key words: Ionospheric forecast, ionospheric storms, neural network, foF2.

\section{Introduction}

Forecasting ionospheric variations including storms has been studied for many years for high-frequency (HF) radio communications and satellite communications. There are two kinds of variations in the ionosphere. One is periodical changes such as daily, seasonal, and solar cycle variations and the other is a sporadic variation caused by solar-origin disturbances. Periodical variation of the ionosphere is important for determining MUF (maximum usable frequency) of HF communication links. Delays in satellite signals passing through the ionosphere also vary according to the total electron content. Sporadic variations of the foF2 can be classified into three categories: ionospheric positive and negative storms, and TID (travelling ionospheric disturbances). Ionospheric storms are drastic changes in the plasma density associated with geomagnetic storms caused by highly variable solar winds and magnetospheric energy inputs to the Earth's upper atmosphere. Increase in plasma density is called a positive storm and decrease in plasma density is called a negative storm. Positive storms induce extra delays in GPS radio waves, whereas negative storms cause HF communication blackouts. In this article, we are not concerned with TIDs, which have a period of several hours, because it is difficult to determine the origin required for forecasting in individual events.

The plasma density of ionosphere depends on the balance of production by photo-ionization and losses by a chemical recombination (Yonezawa, 1966). Thus, the ionosphere shows a variety of periodic fluctuations caused by the changes in the solar flux, season, and local time. Ob-

Copyright (c) The Society of Geomagnetism and Earth, Planetary and Space Sciences (SGEPSS); The Seismological Society of Japan; The Volcanological Society of Japan; The Geodetic Society of Japan; The Japanese Society for Planetary Sciences; TERRAPUB servation of the ionosphere has been carried out over many decades by using ionosondes all over the world including Japan. There are empirical models based on those observations, such as IRI (Bilitza et al., 1993), predicting averaged states of the ionosphere. However, it is not well established to predict day-to-day variations including storms.

An important mechanism of negative ionospheric storms is mixing of the upper and lower atmospheres caused by atmospheric disturbances driven by energy inputs in the polar atmosphere during geomagnetic storm periods (Prölss, 1995; Matuura, 1972). Mixing of the upper atmosphere accelerates the reduction of the ionosphere due to the atmospheric composition changes (Matsushita, 1959) by upwelling of the molecular-rich air, because atomic oxygen (O) contributes the production and molecular nitrogen $\left(\mathrm{N}_{2}\right)$ contributes the reduction of the ionosphere (Torr and Torr, 1979). On the other hand, an important mechanism of positive ionospheric storms at mid latitudes is an uplift of the ionosphere due to the upward $E \times B$ drift by penetration of magnetospheric electric fields (Martyn, 1953) or due to an equatorward thermospheric wind (Jones and Rishbeth, 1971; Lei et al., 2004). The vertical drift due to electric fields or neutral air winds displaces the equilibrium position of the peak to a new level, at which the upward forcing balances with the downward diffusion along the magnetic field line (Rishbeth, 1978). During such uplifts, reduction of the ionosphere by $\mathrm{N}_{2}$ is slowed (Prölss, 1995).

There have been many attempts to predict and forecast foF2 using neural networks (Altinay et al., 1997; Cander and Lamming, 1997; Williscroft and Poole, 1996). Neural networks, a kind of artificial intelligence methods, are widely used to describe complicated non-linear input/output relationships. In particular, multi-perceptron neural networks using the error back propagation algorithm 
are very useful for modelling complicated phenomena because of their ability of non-linear function approximation (Rumelhart et al., 1986). Recently, they are applied to predicting the F-layer parameters in South Africa (McKinnell and Poole, 2004), short-term prediction of foF2 (Wintoft and Cander, 1999), short-term prediction of foF2 in Russia (Barkhatov and Renynov, 2004), temporal and spatial forecasting (Tulunay et al., 1999), foF2 storm forecasting in Europe (Wintoft and Cander, 2000), a global model for foF2 (Oyeyemi et al., 2005a), and global forecasting up to 5 hours in advance (Oyeyemi et al., 2005b). However, there is as yet no practical method to predict daily variations or to forecast ionospheric storms specific to the Japan's area.

In this work, we developed an operational tool for forecasting foF2 values 24 hours ahead at Kokubunji $\left(35^{\circ} \mathrm{N}\right.$, $\left.139^{\circ} \mathrm{E}\right)$, Tokyo using daily activity parameters easy to access. The prediction is executed once a day between 0900 and 1200 JST (0000 and 0300 UT) when the geomagnetic activity index for the previous UT day becomes available. In this regard, there could be several indices describing geomagnetic activity such as, Kp, Ap, Dst, and AE. We chose the $\mathrm{K}$-index issued by Kakioka magnetic observatory, because of its quick availability. To find the most influential local time of the K-index enhancement in ionospheric storm developments, we analyzed past ionospheric storms occurred in a period of 11 years by using a superposed epoch analysis. The result was used to optimize the inputs to the neural network.

\section{Operational Forecast Model}

\subsection{Error back propagation algorithm}

The feed forward neural network with the error back propagation (Rumelhart et al., 1986) is a method mapping multivariable inputs to multivariable outputs. This type of neural network has an ability of nonlinear functional approximation (Kolmogorov, 1957; Funahashi, 1989). That is, the network extracts features from a data set and learns the association between the inputs and the outputs. The error back propagation algorithm is suitable for predicting natural phenomena for which long-term observational data are available. The network consists of three or more layers. They are the input layer, hidden layer(s), and output layer. Each layer consists of units, and units in one layer are connected with units in the next layer with 'weights'. The process of determining the weights is called 'learning'. The time for learning and the performance of the trained network depend on the number of layers and the number of units. There is, however, no conclusive way to determine appropriate numbers of layers and units. Once those numbers and weights are fixed, the target parameters for a new input data set are quickly obtained. The neural network we chose consists of three layers: a pair of input/output layers, and single hidden layer. The output layer has only one unit corresponding to the target parameter of foF2. Details of the input and target parameters are described in next section. The number of units in the hidden layer is determined on a trial-and-error basis. The learning procedure is the same as that described by Haykin (1999).

\subsection{Target}

The aim of our operational model is to provide a useful prediction for a variety of fields of radio application such as HF communication/broadcast and advanced GPS applications. The ionospheric critical frequency, foF 2 , is one of the most important factors describing ionospheric conditions, and it is directly connected with the maximum usable frequency of HF communication links. Total electron content (TEC) that specifies the propagation delay of the GPS signals is another important factor. In the meantime, a data set that covers a sufficiently long period is required for the neural network learning, say several times of the solar cycle period. In this regard, bottomside sounding, which provides foF2, has been conducted for more than 60 years at Kokubunji, Tokyo. On the other hand, a reliable database for TEC is available only after $\sim 1998$ over Japan created by using the GPS Earth Observation Network (GEONET). This is not a sufficient length for the neural network learning. Fortunately, TEC is mostly determined by the maximum electron density of the $\mathrm{F}$ layer, and TEC variations can be inferred from foF2 variations to some extent. Thus, we chose foF 2 as a target parameter of the neural network prediction. The leaning of the network was made by using foF2 data for two solar cycles, and the validation of the learning result was made by using the data from another solar cycle. For the efficiency of the learning, the daily variations are not explicitly learned, but the learning process was individually run for each hour, with only one target parameter.

\subsection{Input parameters}

For an effective prediction of foF2, factors that cause the ionospheric electron density variation must be supplied to the input layer as many as possible. An important requirement for our operational model is the quick availability of the input parameters. There might be two categories of input parameters, one causes periodic changes of the ionosphere and the other does sporadic changes or ionospheric storms. Figure 1 presents an example of long term variability of foF 2 in the top panel along with monthly mean solar activity indices, the sunspot number in the middle panel and the $F_{10.7}$ index in the bottom panel, for the period from 1990 to 2004 . The $F_{10.7}$ index indicates the intensity of radio noise at $2.8 \mathrm{GHz}$ (wave-length: $10.7 \mathrm{~cm}$ ). The sunspot number is the solar activity index having a long history. In the figure, solar cycle, annual, and semiannual variations of foF2 are clearly seen. In addition to those periodic variations, a day-to-day scattering is recognized. Figure 2 expands the foF2 variability for the one year period of 2001 in the bottom panel along with the daily geomagnetic activity index $\sum \mathrm{K}$ in the top panel, where $\sum \mathrm{K}$ is the daily sum of the Kakioka's magnetic disturbance index. Large dropouts of foF 2 from the smoothed seasonal variation can be seen several times. Some of those dropouts are correlated with the geomagnetic activity, but others are not. Details of the input parameters are described below.

2.3.1 Solar cycle variation After several trials, we decided to use both $F_{10.7}$ (http://www.drao-ofr.hia-iha.nrccnrc.gc.ca/icarus/www/daily.html) and the sunspot number (http://sidc.oma.be/) as proxies of the solar activity. In Fig. 1, it is noted that their variations are not exactly the same; for example, the maximum of $F_{10.7}$ in the latter half 

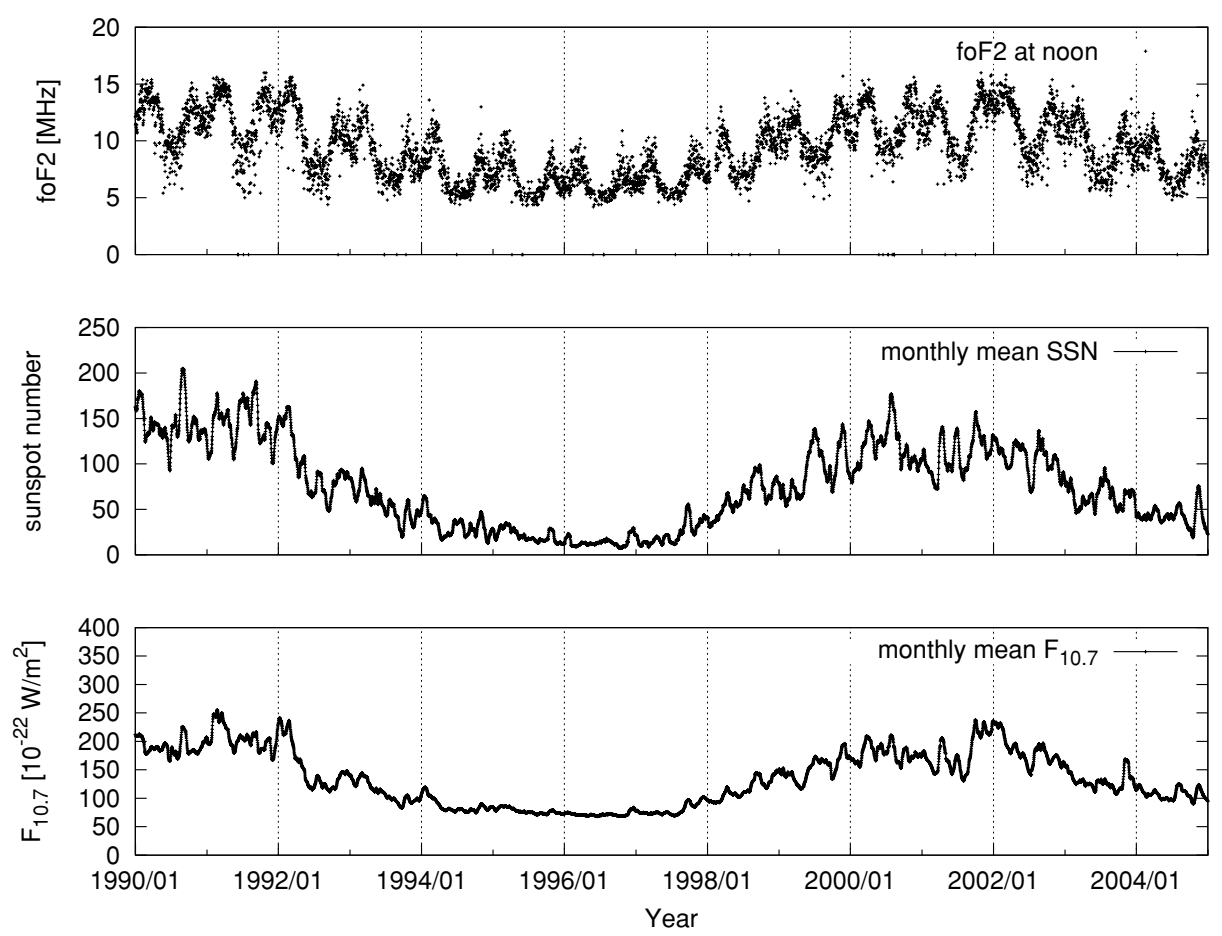

Fig. 1. Variation of foF2, $F_{10.7}$ and SSN for one solar cycle from 1990 to 2005.
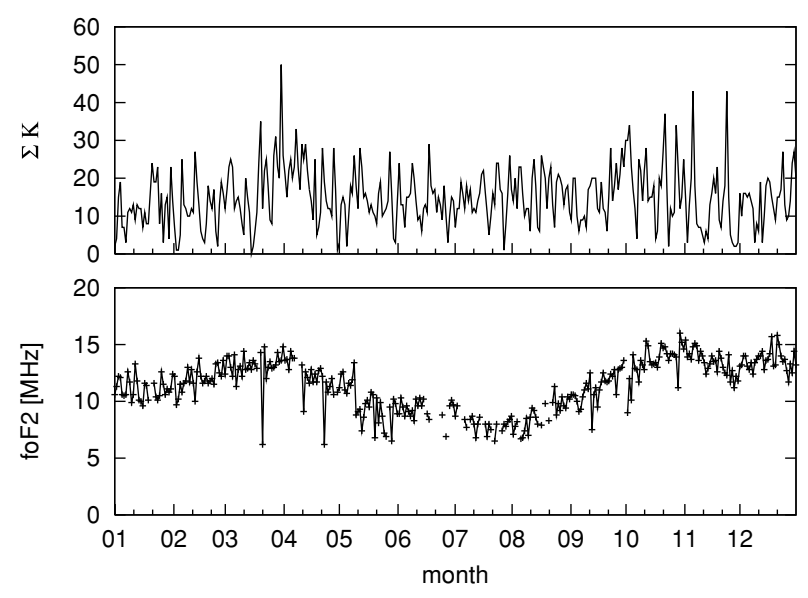

Fig. 2. Variation of $\sum K$ and foF2 for one year in 2001.

of the period appeared in 2002, whereas that of sun spot number did in 2001. As both the daily indices fluctuate too much to learn the neural network efficiently, we took averages over preceding 3 days and the 27-day solar rotational period for $F_{10.7}$ and sunspot number. Therefore, the solar activity are represented by 4 inputs: 3-day and 27-day averages of the solar flux and the sun spot number.

2.3.2 Seasonal variation The day of year (DOY) from 1 to 365 is used to learn seasonal variations. DOY was expressed as a combination of DOY1 and DOY2, both normalized between 0 and 1 , so that the data would continuously change at the end and start of the year as follows.

$$
\mathrm{DOY} 1=\frac{\left(\sin \left(\mathrm{DOY} \cdot \frac{2 \pi}{365}\right)+1\right)}{2}
$$

$$
\begin{gathered}
\mathrm{DOY} 2=\frac{\left(\cos \left(\mathrm{DOY} \cdot \frac{2 \pi}{365}\right)+1\right)}{2} \\
(\mathrm{DOY}=1,2, \cdots 365)
\end{gathered}
$$

2.3.3 Sporadic variation Most non-repeated variations are associated with geomagnetic disturbances. We chose the $\mathrm{K}$-index at Kakioka $\left(36^{\circ} \mathrm{N}, 140^{\circ} \mathrm{E}\right)$, Japan, (http://www.kakioka-jma.go.jp/) which is one of the stations used for derivation of the planetary geomagnetic activity index, Kp. Besides the K-index, there are several geomagnetic indices that represent geomagnetic disturbances, such as $\mathrm{AE}$ (the auroral electrojet index) and $\mathrm{Kp}$ indices. Although the AE index that represents the intensity of energy injection into the polar region is suitable for considering upper atmospheric disturbances, the data do not cover a period long enough for the neural network learning. The planetary $\mathrm{Kp}$ index is derived by calculating a weighted average of $\mathrm{K}$-indices from a network of geomagnetic observatories. Unfortunately, most observatories do not report their data in real-time. However, the K-index of Kakioka is updated daily and is promptly reported on-line. The $\mathrm{K}$ index ranges from 0 to 9 in a quasi-logarithmic scale, and it is related to the most disturbed horizontal magnetic field components relative to a quiet day during a three-hour interval; 8 data are recorded each day. The Kakioka data also cover a sufficiently long period.

For the efficiency of the learning, K-indices were preprocessed based on the analysis of past storm events. Ionospheric storms arise after geomagnetic storm onsets (Matsushita, 1959). However, developments of ionospheric storms depend on the local time of magnetic storm onset and progress after it. To reveal the local time effect on ionospheric storms, superposed epoch analysis was conducted of the K-index variations for significant ionospheric storms. 


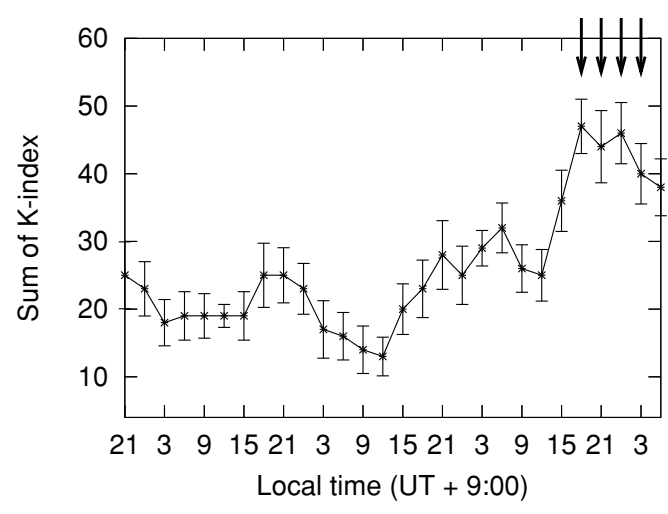

Fig. 3. Superposed epoch analysis of 3-day K-indices variations prior to 10 significant ionospheric storms.

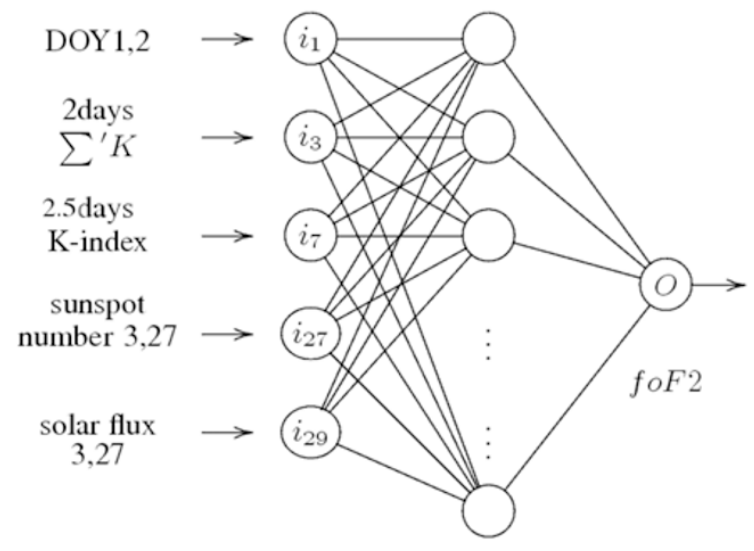

Fig. 4. Neural network (NN) used in this study.

To select such ionospheric storms at Kokubunji, we calculated the deviation of foF2 from the median value (27 days centered at the day considering), $\Delta$ foF 2 , in the period from 1992 to 2003. Thus, we found 10 big negative storms in which the amplitude of $\Delta$ foF 2 exceeded $4 \mathrm{MHz}$. The superposed variation of the K-index is shown in Fig. 3. The figure shows that the fourth to seventh $\mathrm{K}$ indices (down arrows) increased on the day (UT) before the severe ionospheric storm events. In addition, there is a general tendency of increasing in local $\mathrm{K}$ index during night time (Yumura, 1951). Thus, for effective learning of the network, we defined $\sum{ }^{\prime} \mathrm{K}$; the sum of four K-indices from the fourth to seventh indices of the day and from the eighth index of the day to the third of the next day. $\sum{ }^{\prime} \mathrm{K}$ for two preceding days were used as inputs describing geomagnetic activity, in addition to each $\mathrm{K}$ indices for 2.5 days. Accordingly, the magnetic activity input consists of 24 parameters.

\subsection{Structure of the network and learning}

The neural network for predicting foF 2 was constructed as shown in Fig. 4. The input layer had 30 units and the hidden layer had 200 units. The output layer had one unit for the target parameter of foF2. Data set for the learning of the network should cover possible cases of parameter variations including the solar activity and seasons. Thus at least one solar cycle must be covered. In this study, the learning was executed for the data set obtained from 1960 to 1984 , covering two solar cycles. Before learning a variation
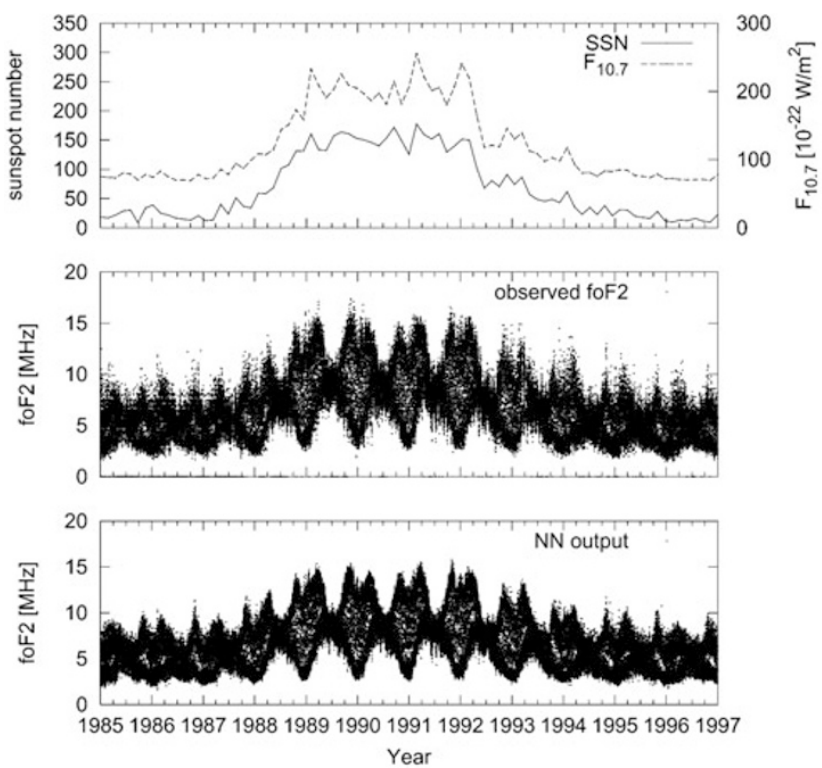

Fig. 5. Comparison of observed foF2 variation (middle panel) and NN prediction (bottom panel) during one solar cycle period from 1986 to 1996. upper panel shows variations of SSN and $F_{10.7}$.

range was examined for each parameters, and input and output data were normalized to have values between 0 and 1 .

\section{Results and Discussion}

Here, we examine the performance of the neural network learning and describe the operational running for daily ionospheric storm forecasting. First, we demonstrate the reproducibility of the periodically repeated variations, then discuss ionospheric storm predictions.

\subsection{Periodic variations}

Validation of the neural network was done using a data set for the one solar cycle period from 1985 to 1996, which was not included in the learning data set. To show an ability of reproducing variations with the 11-year solar cycle, we compared the output from the neural network with the measured foF2. Figure 5 shows hourly foF2 values observed (middle panel) and predicted (bottom panel) by the network along with the daily sunspot number and $F_{10.7}$ solar flux (top panel). In the middle and bottom panels, the envelopes display long term variation of the daily maximum and minimum of foF2. Except for the slight spread for the observed values, daily maximum/minimum envelopes for the observed and predicted foF 2 agree quite well, not only for the solar cycle variation but also for the annual and semiannual variations. Figure 6 shows daily variations for selected weeks representing different seasons from the same data set in Fig. 5. Left three panels are for the solar minimum conditions (1985) and right three panels are for the solar maximum conditions (1992). The blue solid line presents the NN output and the dots are the observed foF 2 . It is clear that the network reproduces the daily variations in any season of the solar minimum and maximum. The prediction errors of the neural network are basically within $1 \mathrm{MHz}$.

Next, we compare the prediction performance of the NN 

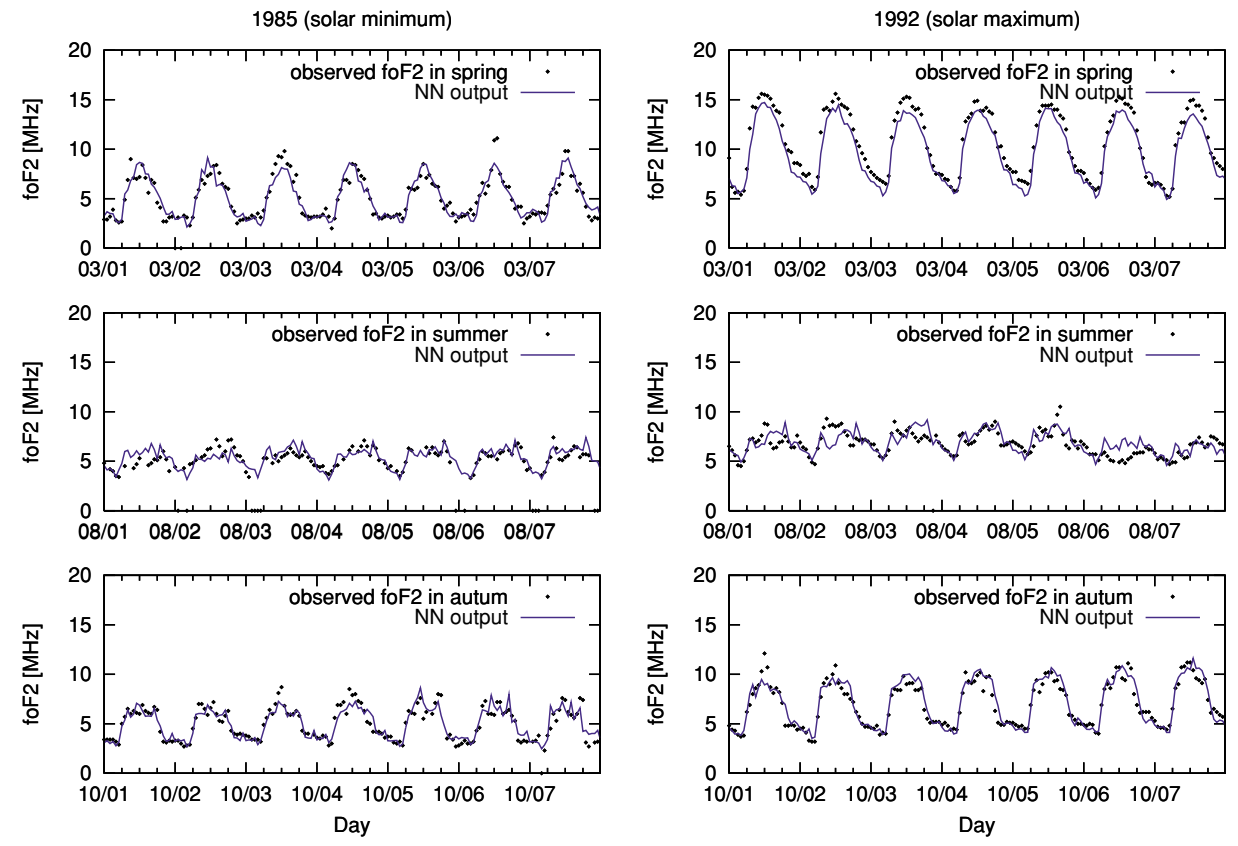

Fig. 6. Comparison of observed foF2 variation (dots) and neural network prediction (blue line) for one week in March, August and October of 1985 (solar minimum: left) and 1992 (solar maximum: right).

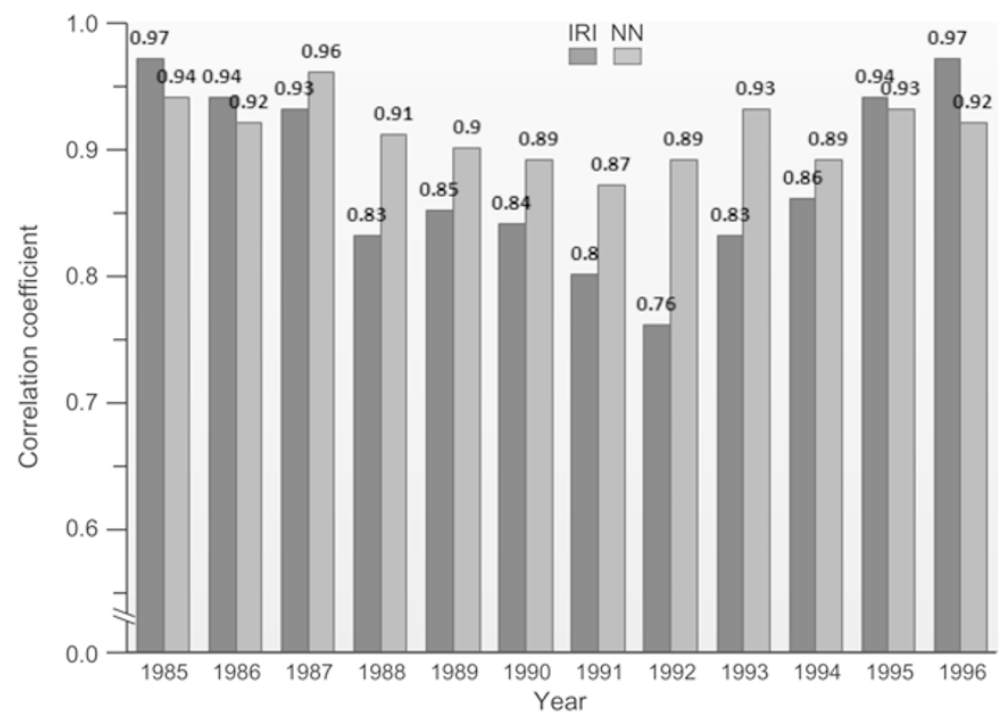

Fig. 7. Correlation coefficients of observed foF2 with NN (light gray) and with IRI (dark gray) in the period from 1985 to 1996.

and that of the empirical ionospheric model, IRI (International Reference Ionosphere). IRI model has tables of ssn and ap-index internal. foF2 was obtained for given years and dates at the location of Kokubunji with standard option. To do this, root mean square errors (RMSE) were calculated for the two predictions. Figure 7 shows the correlation coefficient of the NN output with the observed foF2 (light gray bar) and those of the IRI output with the observed foF2 (dark gray bar). Near the solar maximum (1991-1992), the correlation coefficient for the IRI prediction falls to 0.75 , but that for the NN prediction remains above 0.87 . Near the solar minimum (1985 and 1995-1996), the correlation coefficient for NN were less than that for IRI, although both are above 0.9 . The coefficients averaged over the one solar cycle (1985-1996) are 0.91 for NN and 0.88 for IRI.
We also compared the local time dependence of the performance through the period from 1985 to 1996 in Fig. 8. Figure 8 is the mean errors (MEs) of the IRI (above panels) and NN (below panels). It is noted that the MEs and the deviations for IRI are larger than those for NN and the difference is significant from noon (12 LT) to midnight (0 LT). IRI outputs are relatively high compared to foF2, at Kokubunji especially in solar maximum.

\subsection{Magnetic activity effects}

Our operational forecast model runs once a day just after the beginning of the new UT day (0900 JST) when all input parameters become available, and foF2 values are predicted up to 24 hours in advance. As the input parameters contain magnetic disturbance indices, their ionospheric effect should appear in the predicted foF2 variation, i.e., 
solar maximum (spring + autumn)

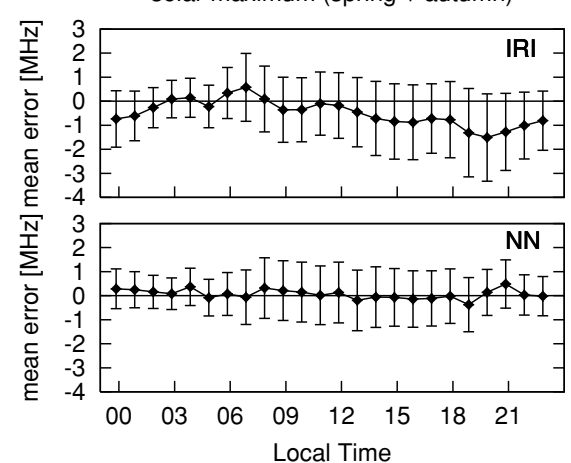

solar minimum (spring + autumn)

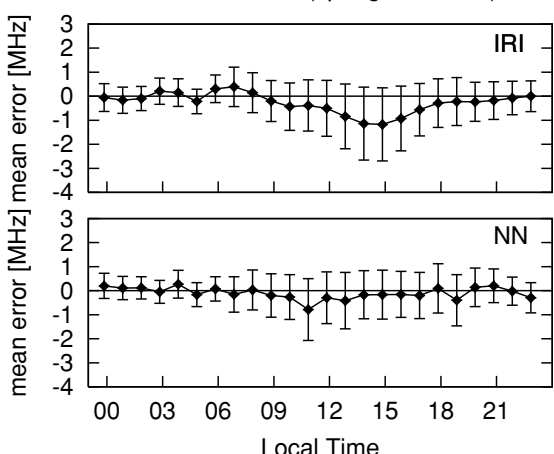

Fig. 8. MEs calculated of NN (gray) and IRI (black) predictions for the period from 1985 to 1996.

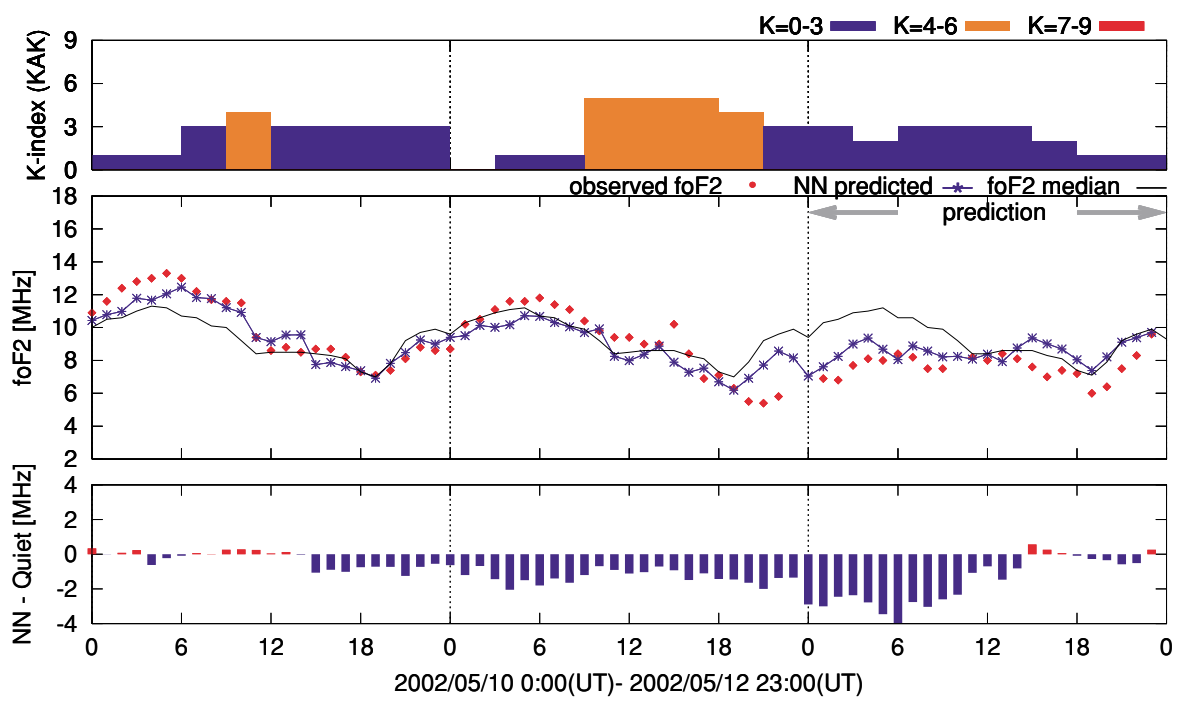

Fig. 9. Ionospheric disturbance for the period from 10 to 12 May 2002. Top panel shows K-index; middle panel compares observed (red dots) and predicted foF2 (blue crosses connected with line), and bottom panel shows predicted ionospheric storm.

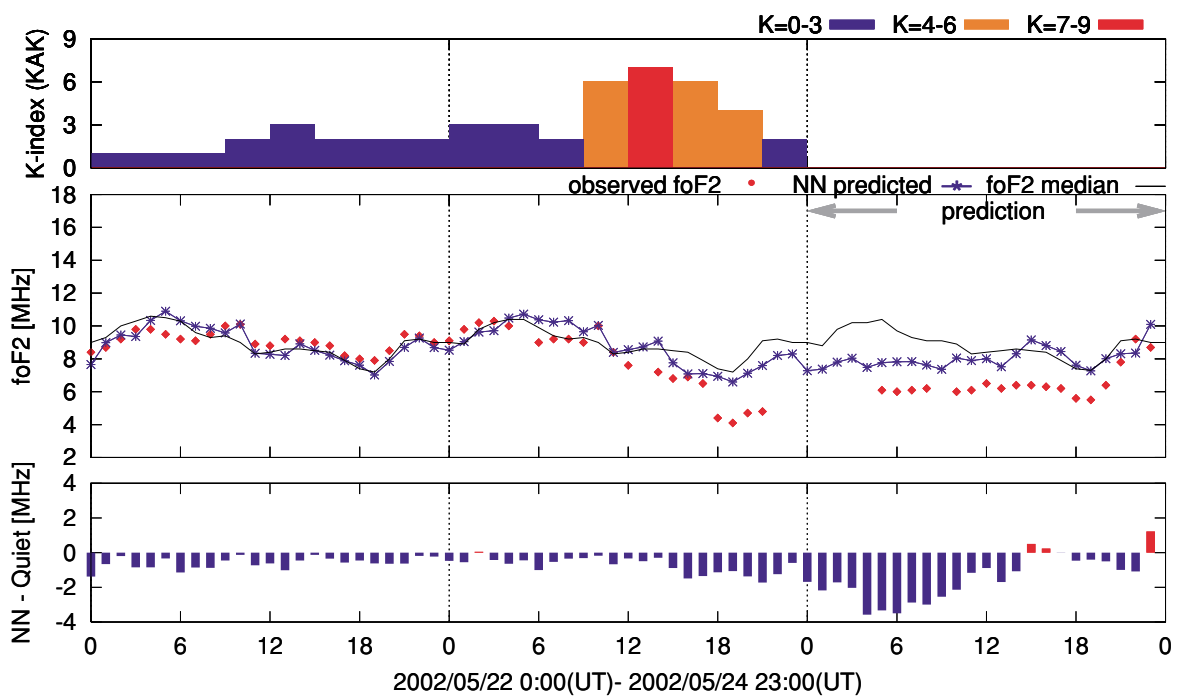

Fig. 10. Same as Fig. 9 but for the period from 22 to 24 May 2002.

ionospheric storms. To evaluate the ability of storm prediction, cases when K-indices largely increased were examined. Top panels in Figs. 9 to 14 are K-indices and the values up to the preceding UT day were used for the pre- diction run. The middle panels show observed (red pluses) and predicted foF 2 (blue crosses connected with line) along with foF 2 median curves (solid lines). The foF 2 median is the median value in the 27 -day period centered on the 


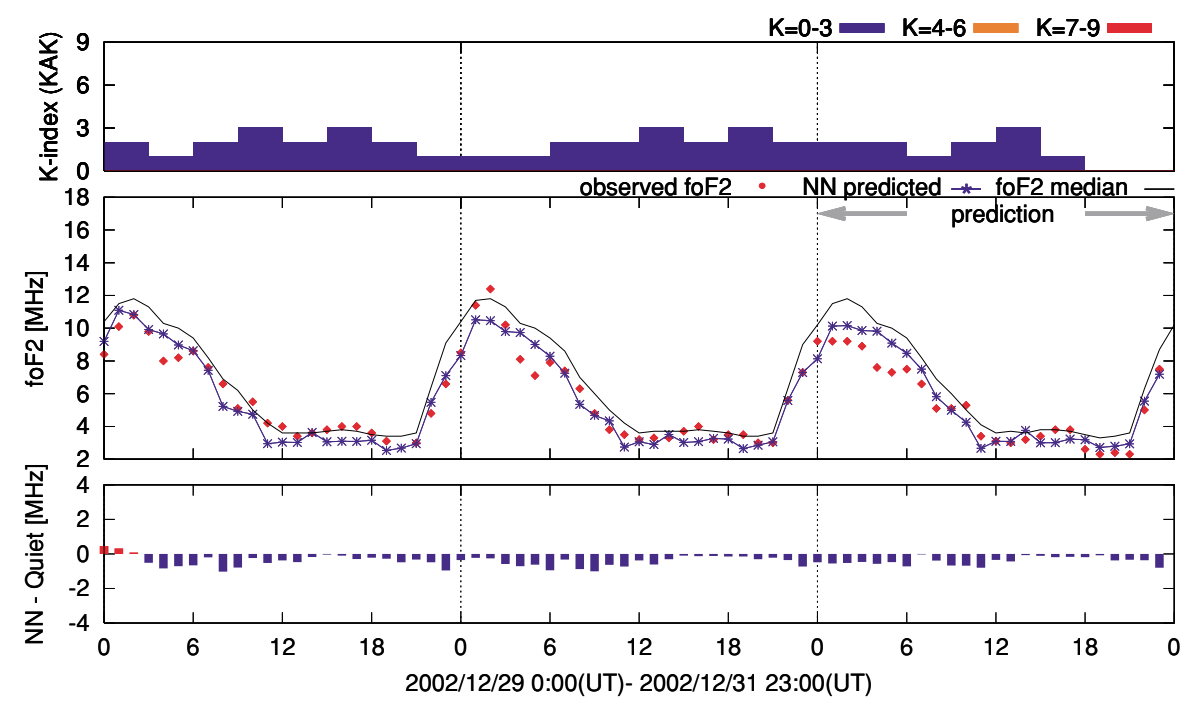

Fig. 11. Same as Fig. 9 but for the period from 29 to 31 December 2002.

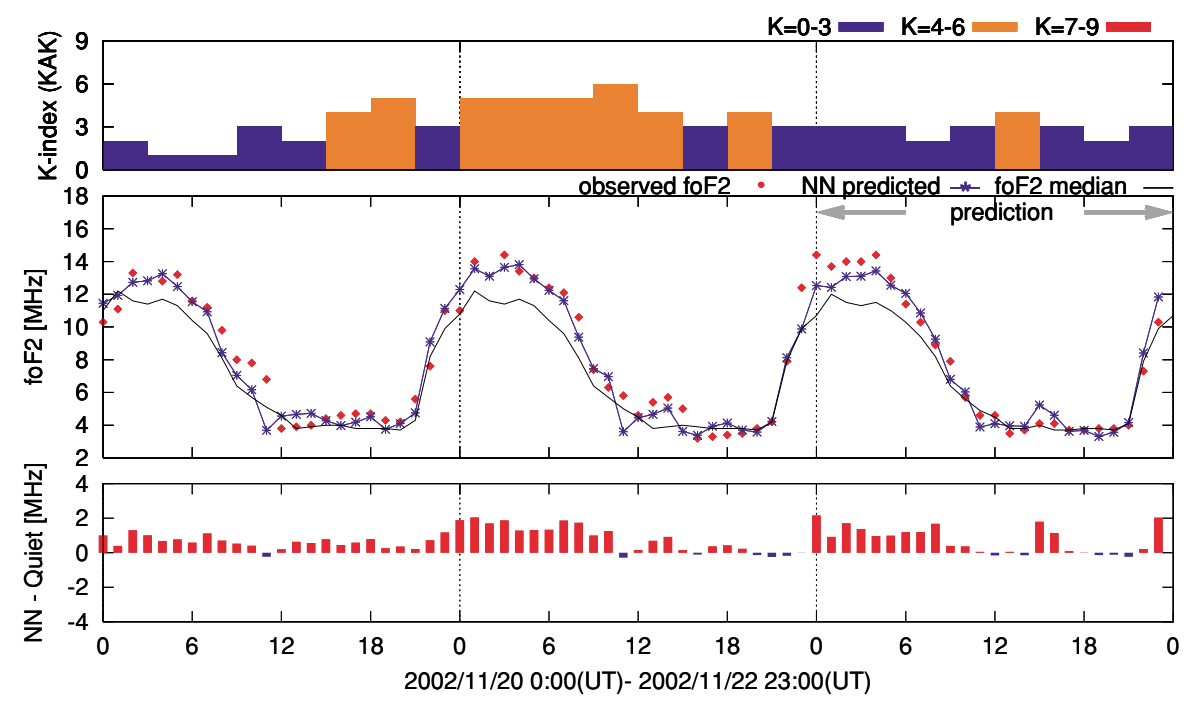

Fig. 12. Same as Fig. 9 but for the period from 20 to 22 November 2002.

day considering. The bottom panels show the difference between the predicted foF 2 and the quiet reference, where the quiet references were produced through another neural network run with all $\mathrm{K}$-indices artificially fixed to 1 , i.e., magnetically quiet condition. Figures 9 to 11 exhibit negative ionospheric storms and Figs. 12 to 14 exhibit positive storms. Individual events are examined below in more detail.

3.2.1 Negative storms In Figs. 9 and 10, the ionospheric negative storm occurred after the geomagnetic disturbance that had occurred one day before. In the example of 22 May 2002, the K-index reached 6 in the 18-20 LT period (fourth K-index of the day) and 7 in the 21-23 LT period (fifth $\mathrm{K}$-index of the day). The large influence of the increase in the $\mathrm{K}$-index in these local times on ionospheric storms is consistent with the preliminary study of superposed epoch analysis (see Fig. 3). Figure 11 presents another storm occurred on 31 December 2002, when moderate geomagnetic disturbances (this term was in a recurrent disturbance period caused by a coronal-hole rotation). Here,
Kakioka Geomagnetic Observatory classifies disturbances with $\sum \mathrm{K}$ from 18 to 23 as a minor storm (Koike, 1991). During 28-31 December 2002, $\sum K$ s are 20,16,16,11 respectively. This example shows that our network can predict such a type of disturbances.

3.2.2 Positive storms In the example of 22 November 2002 (Fig. 12), the K-index reached 5 on 20 November and a modest level continued for the following two days. The ionospheric response to this disturbance was a prolonged positive phase, which were significant during the daytime. The middle (blue crosses connected with line) and bottom (red bars) panels show that the network reproduced the positive variations quite well. Figures 13 and 14 shows another example of positive disturbances, for which geomagnetic storm started on 28 October 2003 and continued for three days. This extreme event is known as the 'Halloween event' caused by a big solar flare (X17.2) at 11:51 UT on 28 October 2003, and subsequently a geomagnetic disturbance occurred at 6:11 UT (15:11 JST) on 29 October. The ionospheric response to this disturbance was 


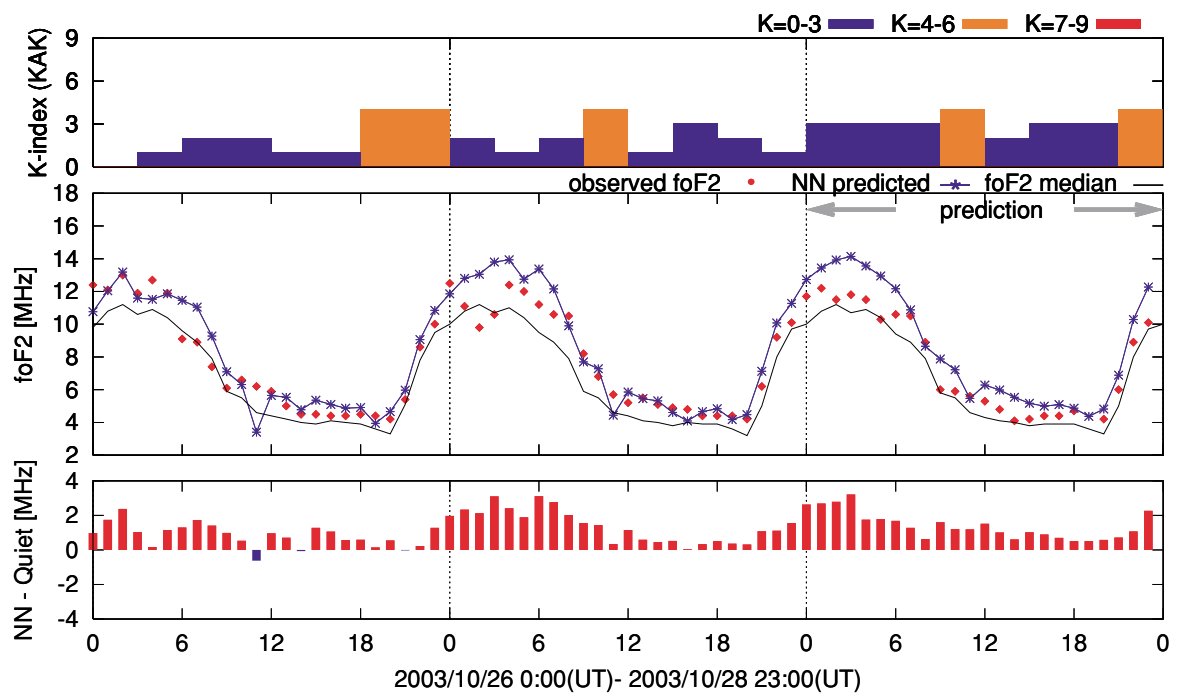

Fig. 13. Same as Fig. 9 but for the period from 26 to 28 October 2003.

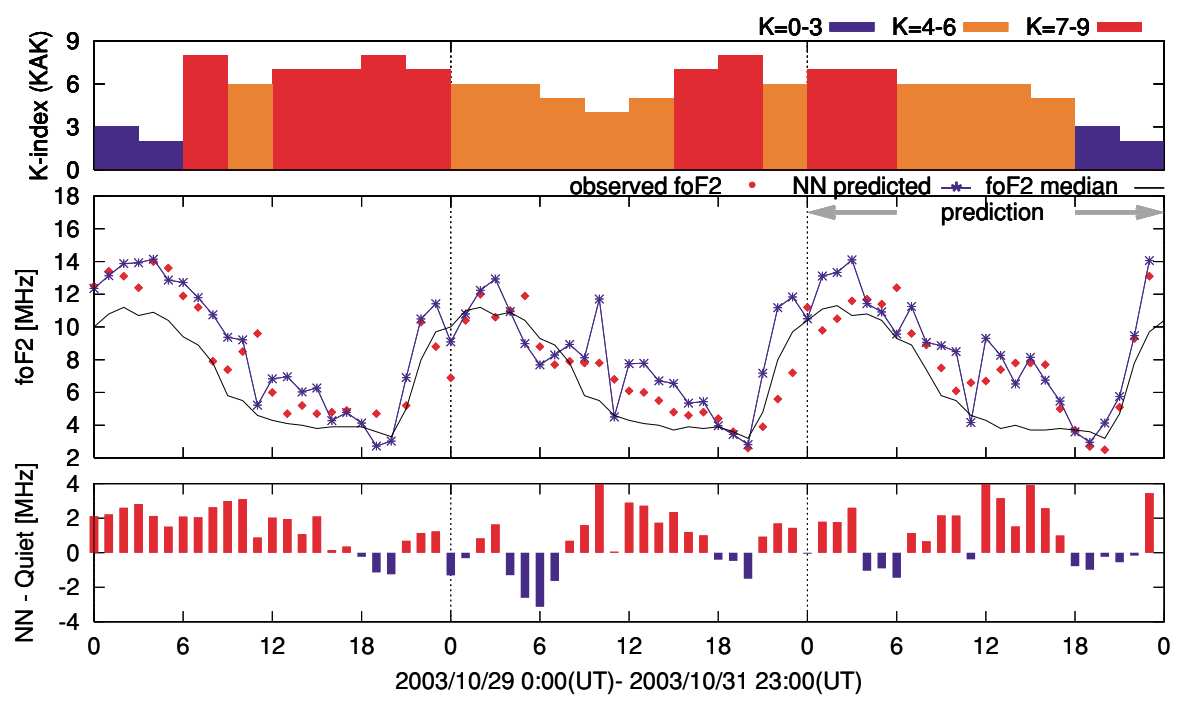

Fig. 14. Same as Fig. 9 but for the period from 29 to 31 October 2003.

quite complex. The network broadly reproduced the disturbances.

3.2.3 Accuracy of storm forecasting In order to examine the total accuracy of storm forecasting, the daily disturbance index $\left(\delta_{\text {obs }}\right)$ was defined as the mean deviation of foF 2 from the quiet reference for 12 hourly-values of foF2 in daytime, because positive disturbances during nighttime are mostly continuation of the preceding day time disturbances and negative storms clearly appear during day time when new ionization process proceeds. For this purpose, the quiet reference was obtained by the NN run with all Kindices fixed to 1 . The ionospheric disturbance was classified into five levels: positive $\left(\delta_{\text {obs }} \geq 1 \mathrm{MHz}\right)$, weak-positive $\left(1>\delta_{\text {obs }}>0.6\right)$, quiet $\left(0.6 \geq \delta_{\text {obs }} \geq-0.6\right)$, weak-negative $\left(0.6>\delta_{\text {obs }}>-1\right)$, and negative $\left(-1 \geq \delta_{\text {obs }}\right)$. Similarly, for the predicted foF 2 the daily disturbance index $\left(\delta_{\mathrm{NN}}\right)$ was determined and was classified into the five levels. Figure 15 shows the score of storm forecasting by $\mathrm{NN}$ for the period from 1985 to 1996 . The boldface numbers mean that ionospheric conditions were successfully forecasted (2488 out of 4383 days). Hatched numbers are the cases forecasts was failed (661 out of 4383 days). The others are poor cases (1234 out of 4383 days). Our NN model predicts ionospheric storms based on the time history of the K-index alone. For more accurate predictions, more parameters such as the storm onset time and the Dst index are required.

\section{Summary}

An operational forecasting model of ionospheric variations and storms for the mid-latitude station, Kokubunji, Japan was constructed. The model is based on a neural network whose inputs are solar indices and a geomagnetic disturbance index. Regarding the geomagnetic indices, we performed a superposed epoch analysis for significant ionospheric storms. The result indicated that the fourth to seventh $\mathrm{K}$-indices of the day are influential on ionospheric storms occurring on the next day. Based on this analysis and diurnal variations of the local $\mathrm{K}$ value, $\sum{ }^{\prime} \mathrm{K}$ was defined as summing up 4 indices. The $\mathrm{NN}$ was trained with these inputs using data set from 1960 to 1984, and was validated 


\begin{tabular}{|c|c|c|c|c|c|}
\hline Obs & Negative & $\begin{array}{c}\text { Weak } \\
\text { Negative }\end{array}$ & Quiet & $\begin{array}{c}\text { Weak } \\
\text { Positive }\end{array}$ & Positive \\
\hline Negative & $\mathbf{4 8}$ & 32 & 112 & 12 & 9 \\
\hline $\begin{array}{c}\text { Weak } \\
\text { Negative }\end{array}$ & 45 & $\mathbf{4 0}$ & $17 \mid$ & 30 & 11 \\
\hline Quiet & 119 & 191 & $\mathbf{2 2 6 2}$ & 254 & 158 \\
\hline $\begin{array}{c}\text { Weak } \\
\text { Positive }\end{array}$ & 10 & 22 & 463 & $\mathbf{8 1}$ & 40 \\
\hline Positive & 3 & 4 & 171 & 38 & $\mathbf{5 7}$ \\
\hline
\end{tabular}

Fig. 15. Accuracy of storm forecast during 1985 to 1996 (4383 days).

with the data set from 1985 to 2003 . The validation shows that our neural network is capable of predicting foF2 with an accuracy better than IRI (see Figs. 7 and 8). Throughout the one solar cycle period (1985-1996), the average of correlation coefficients of the NN prediction with the observations was 0.91 and that of the IRI prediction with the observations was 0.88 . Furthermore, our network can forecast negative storms (see Figs. 9 and 10) and positive storms (see Figs. 12-14) both which occur after geomagnetic disturbances. The network is used not only for daily forecasts of ionosphere but also for long-term prediction by using solar activity predictions.

Acknowledgments. Data were provided by the WDC for Kakioka Magnetic Observatory, Japan Meteorological Agency, the Dominion Radio Astrophysical Observatory in British Columbia, Canada and SIDC, RWC Belgium, the World Data Center for the Sunspot Index, Royal Observatory of Belgium.

\section{References}

Altinay, O., E. Tulunay, and Y. Tulunay, Forecasting of ionospheric critical frequency using neural networks, Geophys. Res. Lett., 24(12), 14671470, 1997.

Barkhatov, N. A. and S. E. Renynov, Forecasting of the critical frequency of the ionosphere F2 layer by the method of artificial neural networks, Int. J. Geomagn. Aeron., 5, GI2010, 2004.

Bilitza, D., K. Rawer, L. Bossy, and T. Gulyaeva, International reference ionosphere-past, present, future, Adv. Space Res., 13, 3-23, 1993.

Cander, L. R. and X. Lamming, Neural networks in ionospheric prediction and short term forecasting, 10th International Conference on Antennas and Propagation, IEEE Conf. Publ., 436, 27-30, 1997.
Funahashi, K., On the approximate realization of continuous mappings by neural networks, Neural Networks, 2, 183-192, 1989.

Haykin, S., Neural networks, a comprehensive foundation, Prentice Hall Inc., Upper Saddle River, NJ, 1999.

Jones, K. L. and H. Rishbeth, The origin of storm increases of mid-latitude F-layer electron concentration, J. Atmos. Terr. Phys., 33, 391-401, 1971.

Koike, K., Statistics of the K-index, Kakioka Magnetic Observatory, Gijutsu Hokoku, 31, 32-46, 1991.

Kolmogorov, A. N., On the representation of continuous functions of many variables by superposition of continuous functions of one variable and addition, Doklady Akademii Nauk SSSR, 144, 679-681, 1957.

Lei J. H., L. B. Liu, W. X. Wan, and S. R. Zhang, Modeling investigation of ionospheric storm effects over Millstone Hill during August 4-5, 1992, Earth Planets Space, 56(9), 903-908, 2004.

Martyn, D. F., The morphology of the ionospheric variations associated with magnetic disturbance, Proc. R. Soci. London, Ser. A, 218, 1-18, 1953.

Matsushita, S., A study of the morphology of ionospheric storms, J. Geophys. Res., 13, 305-321, 1959.

Matuura, N., Theoretical models of ionospheric storms, Space Sci. Rev., 13, 124-189, 1972.

McKinnell, L. A. and A. W. V. Poole, Predicting the ionospheric F layer using neural networks, J. Geophys. Res., 109, doi:10.1029/ 2004JA0104445, 2004.

Oyeyemi, E. O., A. W. V. Poole, and L. A. McKinnell, On the global model for foF2 using neural networks, Radio Sci., 40, doi:10.1029/ 2004RS003223, 2005a.

Oyeyemi, E. O., A. W. V. Poole, and L. A. McKinnell, On the global short-term forecasting of the ionospheric critical frequency foF2 up to 5 hours in advance using neural networks, Radio Sci., 40, doi:10.1029/ 2004RS003239, 2005b.

Prölss, G. W., Ionospheric F-region storms, in Handbook of Atmospheric Electrodynamics, vol. 2, (Ed) Volland, H., edited by Boca Raton, 195248 pp., CRC Press, 1995.

Rishbeth, H., S. Ganguly, and J. C. G. Walker, Field-aligned and fieldperpendicular velocities in the ionospheric F2-layer, J. Atmos. Terr. Phys., 40, 767-784, 1978.

Rumelhart, D. E., G. E. Hinton, and R. J. Williams, Learning representation by back-propagation errors, Nature, 323, 533-536, 1986.

Torr, D. G. and M. R. Torr, Chemistry of the thermosphere and ionosphere, J. Atmos. Terr. Phys., 41, 797-839, 1979.

Tulunay, A. K. E., I. Topalli, and Y. Tulunay, Temporal and spatial forecasting of ionospheric critical frequency using neural networks, Radio Sci., 34, 1497-1506, 1999.

Williscroft, L. A. and A. W. V. Poole, Neural networks, foF2, sunspot number and magnetic activity, Geophys. Res. Lett., 23, 3659-3662, 1996.

Wintoft, P. and Lj. R. Cander, Short-term prediction of foF2 using timedelay neural network, Phys. Chem. Earth, 24, 343-347, 1999.

Wintoft, P. and Lj. R. Cander, Ionospheric foF 2 storm forecasting using neural networks, Phys. Chem. Earth, 25, 267-273, 2000.

Yumura, T., On the 'three-hour-range Indices' K at Kakioka, Memoirs of the Kakioka magnetic observatory, 6, 1-17, 1951.

Yonezawa, T., Theory of formation of the ionosphere, Space Sci. Rev., $\mathbf{5}$, 3-56, 1966.

M. I. Nakamura (e-mail: maho@nict.go.jp), T. Maruyama, and Y. Shidama 